187

\title{
CITOCHINE E VALORI DI RIFERIMENTO
}

Paparella C.; La Mancusa R.; Panetta V.; Spanò A.

Dipartimento di Diagnostica Strumentale:

Laboratorio di Microbiologia Virologia Immunologia

Ospedale Sandro Pertini

Le citochine costituiscono una rete complessa di piccoli mediatori proteici che sono secreti e interagiscono con recettori cellulari ad alta affinità. Agendo all'interno del sistema immune e interagendo anche con altri sistemi dell' organismo formano una rete integrata coinvolta nella regolazione delle risposte immuni.

L'uso di metodiche accurate e sensibili per la misurazione delle citochine nei fluidi biologici è un pre-requisito assoluto per definire l'implicazione di questi mediatori in situazioni cliniche differenti.

Per la quantificazione delle citochine vengono utilizzate metodiche differenti e tra queste la Citometria a flusso rappresenta un approccio efficiente alla misura delle complesse interazioni dei parametri coinvolti nelle risposte immunologiche.

Il sistema CBA è una nuova metodologia analitica che consente la simultanea quantificazione di diversi analiti solubili presenti in un unico campione biologico. Il sistema si basa sull'uso di una serie di particelle (biglie), ciascuna dotata di una intensita' di fluorescenza discreta tale da consentire la rivelazione simultanea dei vari analiti. Tali particelle portano sulla loro superficie l'anticorpo di cattura specifico per l'analita e la metodica consente di ottenere un range dinamico di 3 logaritmi.

Tale tecnologia è stata utilizzata nel nostro laboratorio per comparare la riproducibilità di risultati analizzati con la metodica CBA con risultati provenienti da altre metodiche. In particolare sono stati analizzati 100 campioni di plasma e di siero appartenenti ad una popolazione con bassa prevalenza di patologia ( soggetti donatori afferenti al SIT dell'ospedale Sandro Pertini di Roma ). Tale studio è stato finalizzato alla valutazione dei range di riferimento delle citochine analizzate: IL1b, IL2, IL4, IL5, IL6, IL8, IL10, TNF-a, IFNg, IL-12p70.

Attraverso il calcolo dei percentili è stato possibile individuare i range di riferimento relativi alla nostra popolazione che sono stati riportati e valutati in opportune tabelle. 